\title{
HEMATOMA SUBDURAL AGUDO TRAUMÁTICO
}

\author{
ESTUDO DE 110 PACIENTES
}

\author{
NICANDRO DE FIGUEIREDO NETO*; JOHNWY WESLEY G. MARTINS*, \\ MIGUEL FARAGE FILHO**, LUIZ AUGUSTO CASULARI ROXO DA MOTTA***, \\ PAULO ANDRADE DE MELLL $0^{* * * *, ~ R O N A L D O ~ S E ́ R G I O ~ S A N T A N A ~ P E R E I R A ~ * * ~}$
}

\begin{abstract}
RESUMO - Apresentamos uma série consecutiva de 110 pacientes com hematoma subdural agudo traumático (HSDA) admitidos no serviço de emergência do HBDF no período de $1^{\circ}$-janeiro a $1^{\circ}$-dezembro-1994. Todos os pacientes foram atendidos de acordo com o mesmo protocolo. Houve predominância do sexo masculino (79\%), com idade variando entre 14 e 70 anos, sendo os atropelamentos (34\%) e os acidentes automobilísticos (20\%) as causas mais comuns. A maioria dos pacientes $(85,7 \%)$ foi admitida muito grave, com 8 pontos ou menos na Escala de Coma Glasgow (ECG), o que influenciou diretamente na mortalidade. A tomografia computadorizada de crânio foi o exame diagnóstico de escolha que mostrou serem as contusð̃es e o inchaço cerebral ("swelling") as lesðes intracranianas associadas mais frequentes. A cirurgia foi realizada em $45,1 \%$ dos pacientes, e, em sua maioria, através de craniotomia fronto-têmporo-parietal ampla, com drenagem do hematoma, seguida de plástica da dura-mater. Em $54,9 \%$ as condiçð̄es clínicas não permitiram a realização da cirurgia; neste grupo, cerca de $69,6 \%$ estavam em coma profundo à admissão, com 3 pontos na ECG. A letalidade cirúrgica foi de $61,2 \% \mathrm{e}$ esteve diretamente relacionada à condição clínica inicial e à idade do paciente. A letalidade, incluindo todos os pacientes cirúrgicos e não cirúrgicos com HSDA, mesmo aqueles admitidos já com sinais de falência de tronco cerebral, foi de $79,5 \%$. Além destes pacientes que faleceram, cerca de $7 \%$ evoluíram sem sequelas ou com sequelas minimas; outros $11,4 \%$ com sequelas de moderadas a graves e $2,1 \%$ permaneceram em estado vegetativo persistente. Nossos dados esta de acordo com os da literatura no que se refere a elevada taxa de morbidade $\mathrm{e}$ mortalidade dos pacientes com HSDA.
\end{abstract}

PALAVRAS-CHAVE: traumatismo cranioencefálico, hematoma subdural agudo traumático, epidemiologia, diagnóstico, conduta.

\section{Acute traumatic subdural hacmatomas: study of 110 cases}

ABSTRACT - We report a series of 110 patients with acute traumatic subdural hematoma (ASDH) admitted at HBDF emergency within 1994 (January $1^{\text {st }}$ to December $1^{\mathrm{s}}$ ). All patients were treated according to the same protocol. There was a predominance of males (79\%), with ages ranging from 14 to 70 , being car accidents (20\%) and car-pedestrian accidents (34\%) the most frequent causes The majority of patients $(85.7 \%)$ was admitted in very serious condition, with a score of 8 points on the Glasgow Coma Scale (GCS) or lesser, which directly influenced the mortality rates. CT scan was the diagnostic procedure of choice, and it showed contusion and brain swelling to be the most frequent associated intracranial lesions. Surgery was carried out in $45.1 \%$ of cases and, in most instances, through an ample fronto-temporo-parietal craniotomy, with hematoma drainage and dural reconstitution. In $54.9 \%$ of cases, clinical conditions did not allow surgery and in this group, $69.6 \%$

Unidade de Neurocirurgia do Hospital de Base do Distrito Federal (HBDF): * Médico Residente da Unidade de Neurocirurgia do HBDF; ** Médico staffs e preceptores da Unidade de Neurocirurgia do HBDF; *** Médico staff do HBDF e Professor de Endocrinologia da Universidade de Brasilia; **** Professor de Neurocirurgia da Universidade de Brasilia. Aceite: 23-janeiro-1996. 
were in deep coma on admission, with 3 points on the GCS. Surgical lethality was $61.2 \%$ and was directly related to the initial clinical condition and age of the patient. Lethality, including all surgical and non-surgical patients with ASDH, even those admitted without brain stem reflexes, was $79.5 \%$. Besides these patients who died, about $7 \%$ evolved without sequelae or with minimal ones; another $11.4 \%$ had moderate to serious sequelae and $2.1 \%$ remained in persistent vegetative state. Our data are consistent with those found in the literature referring to high rates of morbidity and mortality of patients with ASDH.

KEY WORDS: cranioencephalic trauma, acute traumatic subdural hematoma, epidemiology, diagnostics, management.

O hematoma subdural agudo (HSDA) ocorre em cerca de 1 a $5 \%$ de todas as lesð̃es traumáticas sobre o crânio ${ }^{7,18}$, e em $22 \%$ dos traumatismos cranioencefálicos (TCE) graves ${ }^{7}$. Apesar de todos os novos conhecimentos em neurotraumatologia, da melhoria das condiçðes de transporte dos pacientes traumatizados e da qualidade de atendimento, com monitorização da pressão intracraniana, e do tratamento mais agressivo, o HSDA continua a ser uma das patologias traumáticas mais letais. São descritas elevadas taxas de letalidade, que variam de 50 a $90 \%$ na maioria das séries da literatura ${ }^{1,5,11,13,19,20,33}$.

Vários fatores influenciam significativamente o prognóstico destes pacientes. Um destes fatores é a idade. Tem sido referido que os mais jovens evoluem melhor que os adultos após TCE, apresentando taxa de morbi-mortalidade inferior em relação àqueles com idade mais avançada ${ }^{3,17,20,22,27,32}$. Outro importante fator prognóstico é o estado de consciência do paciente à admissão: os pacientes comatosos evoluem com mortalidade cerca de 5 vezes maior em relação aos pacientes conscientes ${ }^{17,19,22,23,27,32}$. Alguns estudos mostram que a drenagem do hematoma precocemente, dentro das primeiras 4 horas, reduz a mortalidade ${ }^{15,17,28,29,32}$, embora seja ainda assunto controverso ${ }^{8,19,20,36}$. No presente estudo fazemos uma avaliação deste grupo de pacientes e dos fatores prognósticos em relação d letalidade.

\section{MATERIAL E MÉTODOS}

Foram analisados 110 pacientes consecutivos com HSDA, admitidos no setor de politraumatizados do HBDF, no periodo compreendido entre $1^{\circ}$-janeiro a $1^{\circ}$-dezembro-1994. Foram incluidos todos os pacientes com TCE, cuja tomografia de crânio, arteriografia cerebral ou necrópsia confirmaram a presença de hematoma no espaço subdural, independentemente do número de pontos na escala de coma de Glasgow (ECG) à admissão. A maioria dos pacientes foi acompanhada prospectivamente; alguns dados foram coletados dos prontuários e dos achados de necrópsia, no caso dos pacientes que evoluíram para o óbito.

Todos os pacientes foram atendidos inicialmente na sala de emergência do setor de politraumatizados do HBDF, por equipe multidisciplinar e avaliados pelo neurocirurgião. Nela foram levadas a efeito as condutas gerais de estabilização clínica e a avaliaçđo neurológica. Após os cuidados iniciais de ressuscitaçăo, os pacientes foram levados à investigaçăo neuroradiológica, preferencialmente a tomografia computadorizada de crânio (TCC), excetuando-se aqueles com 3 pontos na ECG que não responderam às manobras de ressuscitação. Pacientes cujas TCC mostravam presença de coleção subdural, causando efeito de massa com desvio da linha média, foram levados ao centro cirúrgico para drenagem do hematoma e posteriormente conduzidos à Unidade de Terapia Intensiva ou de Neurocirurgia. A maioria dos pacientes foi submetida a craniotomia descompressiva ampla, fronto-têmporo-parietal, associada a craniectomia da fossa temporal, abertura da dura-máter em estrela, seguida de plástica da dura-máter com periósteo, sendo o "flap" ósseo deixado sem fixação ou apenas com fixação central (Becker e col., citado por Youmans ${ }^{37}$ ). Todos os pacientes operados receberam cuidados clínicos agressivos, que consistiram de hiperventilação otimizada, sedação e manitol, baseando-se no quadro clínico e, em grande parte dos pacientes, na monitorizaçđo da pressão intracraniana com cateter subdural ou intraventricular, extração cerebral de oxigênio através da medida da diferença arterio-jugular e pressão de perfusão cerebral.

\section{RESULTADOS}

A maioria dos pacientes era do sexo masculino (79\%). A idade variou na faixa da $2^{a}$ à $7^{a}$ década com pico entre a $3^{a}$ e $5^{\mathrm{a}}$ décadas, sendo que $63 \%$ dos pacientes tinham entre 14 e 40 anos de idade. Conforme apresentado na Tabela 1 , os acidentes que mais contribuíram para a formação do 
Tabela 1. Etiologia do HSDA.

\begin{tabular}{lc}
\hline Tipo de acidente & (N) $\%$ \\
\hline Atropelamento & $(38) 34,54$ \\
Queda & $(28) 25,45$ \\
Acidente automobilístico & (22) 20 \\
Acidente Ciclistico & (8) 7,27 \\
Agressão & (7) 6,36 \\
Outros & (7) 6,36 \\
\hline
\end{tabular}

Tabela 2. Escala de Coma de Glasgow à admissão.

\begin{tabular}{lll}
\hline TCE & ECG & (N) $\%$ \\
\hline leve & $13-15$ & (11) 10 \\
moderado & $9-12$ & (17) 15,45 \\
grave & $3-8$ & (82) 74,54 \\
\hline
\end{tabular}

Obs - 60,3\% dos TCEs graves com ECG 3 e 4.

hematoma foram os de trânsito com $54 \%$ do total, aqui incluidos os atropelamentos e os acidentes automobilisticos.

Sob o ponto de vista clínico, $74,54 \%$ dos pacientes foram admitidos com TCE grave, com ECG menor ou igual a 8. Destes, 60,3\% tinham ECG entre 3 e 4 (Tabela 2). A TCC, exame de escolha para o diagnóstico do HSDA, foi realizada em $68 \%$ dos pacientes e a angiografia carotidea foi utilizada em apenas $3 \%$ dos casos, nas ocasiðes em que a TCC não pode ser executada. A maioria dos pacientes restantes, em que não foi realizado exame radiológico, foi representada por aqueles que se encontravam com ECG de 3 e que não responderam às manobras de ressuscitação.

A maioria dos pacientes apresentava lesões unilaterais e supratentoriais (Tabela 3). As lesões intracranianas associadas mais frequentes foram as contusőes e o inchaço cerebral, como apresentado na Tabela 4.

Cerca de $45,1 \%$ dos pacientes foram submetidos a drenagem cirúrgica do hematoma, conforme descrito em Material e Métodos. Entre os pacientes que não foram operados, a maioria (69,6\%) encontrava-se extremamente grave à admissão $(E C G=3)$ e seu estado não respondeu ou deteriorouse após as medidas iniciais. Em muitos deles, o hematoma só foi demonstrado à necrópsia. Os restantes $30,4 \%$ dos pacientes que não foram levados à cirurgia, apresentavam hematomas laminares pequenos, com pouco efeito de massa, e em alguns o estado era grave devido a outras lesðes associadas.

A mortalidade variou em função da idade e das condições clínicas dos pacientes à admissão (Tabela 5) 0 intervalo de tempo desde a injúria até a cirurgia que pode ser determinado em cerca de $2 / 3$ dos casos, não demonstrou alteração estatisticamente significativa com relação à mortalidade, comparando-se os pacientes operados antes ou após o intervalo de 4 horas do acidente. Em nosso estudo, 79,5\% dos pacientes que apresentaram HSDA evoluíram para o óbito, incluindo aqueles pacientes que chegaram muito graves $(E C G=3)$ e aqueles com sinais clínicos de morte encefálica. A maior taxa de letalidade ocorreu nos pacientes com ECG menor ou igual a 8, sendo que $76,5 \%$ das

Tabela 3. Topografia do HSDA.

\begin{tabular}{lc}
\hline Topografia & (N) $\%$ \\
\hline Hemisfério cerebral direito & $(45) 40,9$ \\
Hemisfério cerebral esquerdo & $(40) 36,36$ \\
Bilateral & $(10) 9,09$ \\
Fossa posterior & $(9) 8,18$ \\
Inter-hemisférico & $(6) 5,45$ \\
\hline
\end{tabular}

Tabela 4. Lesões intracranianas associadas ao HSDA.

\begin{tabular}{ll}
\hline Lesão Intracraniana & (N) \%
\end{tabular}

Contusão/hematoma intracerebral

(40) 36,36

"Swelling"

(38) 34,54

Hematoma extra-dural agudo

(14) 12,7

Outras

(11) 10 
Tabela S. Letalidade relacionada à gravidade do TCE.*

\begin{tabular}{lcccc}
\hline TCE & \multicolumn{2}{c}{ Pacientes } & \multicolumn{2}{c}{ Óbitos } \\
& $(\mathrm{N})$ & $\%$ & $(\mathrm{~N})$ & $\%$ \\
\hline Leve / moderado & $(28)$ & 25,45 & $(16)$ & 58,27 \\
Grave & $(82)$ & 74,54 & $(67)$ & 81,70 \\
\hline
\end{tabular}

$* p<0,05$. mortes ocorreram na 1" semana do trauma. No grupo de pacientes operados, a letalidade foi de $61,2 \%$. A letalidade dos pacientes entre 14 e 40 anos foi de $59,5 \%$ e, nos maiores de 50 anos, de $90 \%(p<0,05)$.

Cerca de $7 \%$ dos pacientes evoluíram com sequelas minimas ou sem sequelas, $11,4 \%$ com sequelas moderadas a graves e $2,1 \%$ com estado vegetativo persistente.

\section{DISCUSSÃO}

OHSDA é achado comum em pacientes que sofreram TCE severo e continua a ser um desafio dificil para o neurocirurgião devido a sua alta morbi-mortalidade. Na formação do HSDA estão envolvidos mecanismos relacionados aos movimentos inerciais de aceleraçăo ou desaceleração rápida, que levam ao cisalhamento de veias pontes da convexidade dos hemisférios cerebrais ${ }^{7,9,13,37}$. Estes movimentos inerciais rápidos e de curta duração tendem a ser produzidos principalmente por quedas e agressðes, enquanto os acidentes automobilísticos provocam movimentos mais lentos em decorrência das propriedades de amortecimento dos choques dos veículos que vão dar origem a outros tipos de lesões, como a lesão axonal difusa, a menos que a velocidade envolvida nos acidentes seja exageradamente alta. O HSDA pode também surgir secundariamente a contusão ou laceração cerebral, em contiguidade com o espaço subdural, ou ocorrer devido a sangramento de origem arterial causado por ruptura de artérias superficiais em consequencia de traumas diretos sobre o crânio. $O$ sangramento de origem arterial pode ocorrer em até um terço dos casos em que se identifica o ponto de hemorragia e, segundo Candon ${ }^{4}$, o sangramento arterial foi encontrado em $50 \%$ dos seus pacientes à cirurgia. $\mathrm{Na}$ fossa posterior, os principais mecanismos são os relacionados aos cisalhamentos de veias pontes, lesão de seios venosos ou tenda do cerebelo e contusão cerebelar ${ }^{7,34,37}$.

Em nossa casuística houve predomínio dos acidentes de trânsito em relação às quedas $\mathrm{e}$ agressões, contrariamente à maioria das séries da literatura, como a de Gennarelli e Thibault ${ }^{13}$. Essa diferença provavelmente se deve ao alto índice de acidentes automobilisticos em Brasilia, fato este provavelmente favorecido pela característica arquitetônica da cidade que possui amplas avenidas sem cruzamentos e que possibilitam altas velocidades, além do desrespeito às leis de trânsito, e da precária segurança dos nossos veículos.

Da mesma forma, a faixa etária dos pacientes acometidos de HSDA que tende a ser mais elevada, de acordo com outros estudos, como os de Seelig ${ }^{29}$ e Stone ${ }^{32}$ não foi confirmada em nosso material. Neste, $63 \%$ dos pacientes tinham idade entre 14 e 40 anos, o que pode decorrer da baixa faixa etária da nossa população e dos tipos de acidentes relacionados.

A apresentação clínica e a evolução dos pacientes com HSDA é variável e inespecifica para o tipo de lesão, podendo muitas vezes apenas auxiliar na respectiva localização. Os sinais pupilares de dilatação ipsilateral e de déficit motor contralateral indicam o lado da lesão hemisférica supratentorial. Todavia, o falso sinal pupilar, devido a trauma ocular ou trauma direto sobre o nervo ótico ou mesencéfalo, pode estar presente em cerca de $21 \%$ dos casos, enquanto o déficit motor homolateral pode ser encontrado em aproximadamente $26 \%$ dos pacientes com $\mathrm{HSDA}^{7}$. Geralmente os pacientes são admitidos em estado grave e, de acordo com Stone ${ }^{32}$, metade dos pacientes se encontram inconscientes desde o momento do TCE. Na nossa série em sua maioria $(74,54 \%)$, os pacientes foram admitidos com TCE grave, refletindo a gravidade dos acidentes a que foram submetidos.

A TCC é o exame de escolha no diagnóstico das lesðes traumáticas intracranianas pelo seu baixo grau de invasividade, rapidez e alta resolubilidade. No HSDA o aspecto típico é a imagem hiperdensa côncavo-convexa extra-axial (em crescente), com efeito de massa significativo sobre o parênquima cerebral. O HSDA pode se apresentar biconvexo semelhante ao hematoma extradural 
agudo (HEDA), geralmente quando produzido por sangramento arterial hiper-agudo, quando associado a um HEDA ou quando houver aderências prévias subdurais. $O$ hematoma, na fase aguda, pode ser isodenso em relação ao tecido em pacientes anemiados ou quando há mistura de sangue e líquor no espaço subdural. Nestas situaçðes pode ser suspeitado pelo efeito de massa por ele produzido e melhor visualizado na TCC com contraste ${ }^{7,37}$. No presente estudo a TCC foi realizada na maioria dos casos $(61,5 \%)$, não sendo realizada apenas quando não disponível ou naqueles casos em que os pacientes eram admitidos em extrema gravidade $(E C G=3)$ e que não responderam às manobras de ressuscitação. A angiografia cerebral não está mais indicada na rotina de investigação de lesões traumáticas, porém este método diagnóstico ainda tem seu papel em situações e serviços onde não se dispõe de TCC.

A cirurgia é o tratamento de escolha para a maioria dos pacientes com HSDA, visando à drenagem do hematoma e a descompressão do tecido nervoso. A técnica cirúrgica empregada atualmente é uma ampla craniotomia fronto-têmporo-parietal descompressiva, ipsilateral à lesăo, com craniectomia da fossa temporal, seguida de abertura ampla da dura-máter em estrela, drenagem do hematoma e colocação de enxerto na dura-máter de modo a deixá-la frouxa, recolocando o osso sem muita fixação. Os nossos pacientes submetidos à cirurgia foram operados mediante esta técnica.

O HSDA pequeno e com pouco efeito de massa pode ser conduzido conservadoramente, através de controle clínico e radiológico cuidadoso. Estudos de Croce e col. ${ }^{8}$ mostraram não haver beneficio em evacuar-se hematomas pequenos $(<\mathrm{ou}=\mathrm{a} 1 \mathrm{~cm})$ e com efeito de massa mínimo, desde que não haja evidencia de hipertensão intracraniana ou déficit neurológico significativo. Segundo autores citados por Schmidek ${ }^{30}$, hematomas menores ou iguais a $3 \mathrm{~mm}$, associados a "swelling" hemisférico e grande desvio da linha média, não tendem a melhorar clinicamente ou reduzir a pressão intracraniana com a descompressão cirúrgica, sendo melhor conduzidos conservadoramente.

Tem-se discutido em relação ao tempo ideal para se fazer a intervenção cirúrgica. Isto porque, no HSDA, além do efeito de compressão local do tecido nervoso e o aumento generalizado da pressão intracraniana, é possível que a liberação de substâncias do próprio hematoma desencadeiem eventos fisiopatológicos que favoreçam a formação de isquemia cerebral. Estes fatos resultariam em edema citotóxico e inchaço cerebral ("swelling") e consequente aumento progressivo da pressão intracraniana, mesmo após a remoção do coágulo ${ }^{24,25}$. Estudos de Miller $^{25}$ demonstram este fato, visto que danos isquêmicos são observados em associação a hematomas minimos, sem efeito de massa, insuficientes para explicar a isquemia cerebral encontrada. Estudos de Mendelow e col. ${ }^{24}$, em modelos experimentais de hematomas em ratos, mostraram que a isquemia provocada pela injeçăo intracerebral de sangue autólogo era significativamente maior que a produzida por volume equivalente de óleo ou de microbalão. A bioquímica da toxicidade do sangue no espaço subaracnoide, tecido cerebral e espaço subdural merece mais estudos, porém há dados interessantes que associam a liberação de ferro com a subsequente produção de radicais livres de oxigênio nocivos ao tecido nervoso ${ }^{2}$. Em nossa série, o inchaço cerebral esteve presente em $35 \%$ dos casos associados ao HSDA.

De acordo com essas observações, tem sido proposto cirurgia precoce nestes pacientes. Seelig ${ }^{29}$ e seus colaboradores mostraram que a intervenção cirúrgica realizada nas primeiras 4 horas reduziu significativamente a mortalidade e a morbidade dos pacientes. Porém estes resultados não foram reproduzidos por outros autores $s^{8,19,20,36}$, que demonstraram não haver diferença estatísticamente significativa quanto ao resultado, se a cirurgia fosse realizada mais precoce ou mais tardiamente. Nossos dados estão de acordo com estes autores já que não tivemos diferença nos resultados entre pacientes operados precocemente, até 4 horas do evento, e aqueles operados após este periodo.

O prognóstico dos pacientes com HSDA é geralmente muito desfavorável e pode ser influenciado pela idade, mecanismos de lesão, condicão clinica à admissão e controle pós-operatório da pressão intracraniana. Diversos autores têm demonstrado que a mortalidade é significativamente menor em pacientes mais jovens ${ }^{3.17,20.22 .27,32}$. Kotwica e Brzezinhki ${ }^{20}$ mostraram uma mortalidade de $25 \%$ no grupo de faixa etária de 18 a 30 anos contra $80 \%$ no grupo de pacientes com idade superior 
a 50 anos. Stone ${ }^{32}$, que correlaciona morbidade e faixa etária, observou recuperação funcional de cerca de $30 \%$ para cada década abaixo de 50 anos. Existem várias possiveis explicą̧ōes para o pobre prognóstico encontrado nos pacientes idosos e, talvez, o fator mais importante a se considerar seja a possibilidade de que cérebros dos idosos tenham sua capacidade de regeneração comprometida, conforme demonstrado por Long ${ }^{21}$. Estudos experimentais, como o de Whittemore e col..$^{35}$, têm demonstrado que padrões temporais e a quantidade de fatores neurotróficos liberados após trauma de crânio em ratos modificam-se significativamente com a idade.

Os mecanismos de lesão mais comuns nos adultos jovens são os acidentes de veículos em alta velocidade, que levam a lesão cerebral direta com relativo pequeno volume do hematoma fazendo com que os jovens se beneficiem mais das medidas gerais de entubação precoce, manutenção da pressão de perfusão cerebral e oxigenação. Estas medidas clínicas, em teoria, irăo oferecer tratamento eficaz e imediato para as lesð̃es cerebrais que ocorrem imediatamente após o impacto ${ }^{17}$. Isto talvez se deva ao fato de que o principal processo patológico em muitos pacientes jovens, seja a lesão parenquimatosa imediata e direta, mais do que o volume do hematoma. Por outro lado, nos pacientes idosos a história clinica é completamente diferente. Neles os mecanismos de lesão mais comuns são as quedas, que são seguidas por intervalo lúcido e, a seguir, por deterioração neurológica. Ao contrário dos jovens, nos idosos o volume de massa do HSDA parece ser o processo fisiopatológico mais importante em relação ao prognóstico. A atrofia cerebral relacionada à idade causa estiramento de veias pontes, o que é citado como um fator contribuinte na predisposição dos idosos em desenvolverem HSDA. Isto faz com que o volume dos HSDA seja 4 vezes maior, em média, causando desvios da linha média 2 vezes maiores, o que proporciona um caráter mais destrutivo e letal do que nos jovens. Portanto, nos idosos a cirurgia para a drenagem do hematoma é o aspecto mais importante do tratamento, segundo Howard e col."

A intensidade do impacto e a presença de contusão ou inchaço cerebral, laceração, lesão axonal difusa, lesões de tronco cerebral e a presença de fragmentos ósseos no parênquima são fatores determinantes quanto ao prognóstico, além das lesões secundárias como a hipóxia, edema, isquemia e a hipertensão intracraniana. As lesð̌es extracranianas associadas, como a raquimedular, tóraco-abdominal, distúrbios hemodinâmicos, entre outras, também podem influenciar na evoluçăo desses doentes.

Os pacientes admitidos em coma, tendem a ter pior prognóstico que aqueles com melhores níveis de consciência ${ }^{17,19,22,23,27,32}$. Estudo de Janieson e Yelland ${ }^{18}$ mostrou que pacientes que se encontravam conscientes no pré-operatório tiveram taxa de mortalidade menor que $10 \%$, enquanto em pacientes inconscientes a taxa de mortalidade foi 4 a 6 vezes maior. A pressão intracraniana refratária ao tratamento representa fator de piora do prognóstico. Geralmente, pressões acima de 20 $\mathrm{mmHg}$ levam a alteraçðes metabólicas neuronais com maior consumo de $\mathrm{O} 2$, aumento da resistência vascular cerebral e diminuição do fluxo sanguíneo cerebral, o que pode causar hipóxia e agravar o edema cerebral associado e, assim, piorar o prognóstico do paciente.

Agradecimentos - Ao Dr. Mário Zan Morello e ao Sr. João Arruda Falcão pela importante colaboraçăo no preparo do manuscrito.

\section{REFERÊNCIAS}

1. Alberico AM, Ward JD, Choi SC, Marmarou A, Young Mf. Outcome after servere head injury: relationship to mass lesion, diffuse injury and ICP course in pediatric and adult patient. $J$ Neurosurg 1987;67:648-656.

2. Becker D.P.Comentário de: Miller JD, Bullock R, Graham DI et al. Ischemic brain damage in a model of acute subdural hematoma. Neurosurgery. 1990;27,433-439.

3. Cagetti $B$, Cossu M, Pau A et al. The outcome from acute subdural and epidural hematoma in very elderly patients. Br J Neurosurg 1992;6:227-231.

4. Candon $\mathrm{E}$. Indentation sign in the sylvian sulcus and the arterial origin of an acute subdural hematoma: contribution of computerized tomography. Ann Radiol (Paris) 1993;36:269-274.

5. Cantore GP, Delfini R, Neri J.Contribution to the surgical treatment of acute subdural hematomas. Acta Neurochir (Wien) 1987;41:349-353. 
6. Ciembroniewicz JE. Subdural hematoma of the posterior fossa: review literature with additional 3 cases. J Neurosurg 1965;22:465-473.

7. Cooper RP. Post-traumatic intracranial mass lesion. Head injury. Ed 2. Baltimore: Willians \& Wilkins, 1987;238-284.

8. Croce MA, Dent DL, Menke PG, Robertson JT, Hison MS, Young BH, Donovan TB, Pritchard FE, Minard G, Kudsk KA. Acute subdural hematoma: nonsurgical management of selected patients. J Trauma. 1994;36: 820-826.

9. Eisenberg MH, Aldrich EF. Extra-cerebral lesions. Management of head injury. Neurosurg Clin N Am 1991;358-632.

10. Estridge MN. Acute subdural hemorrage of posterior fossa: report of a case and review of the literature. J Neurosurg 1961;18:248-249.

11. Fell DA, Fitzgerald S, Moiel RH, Caram P. Acute subdural hematoma: review of 144 cases. J Neurosurg 1975;42:37-42.

12. Gennarelli TA, Spielman GM, Langfitt TW, Gildenberg PL, Harrington T, Jane JA, Marshall JD, Pitts LH. Influence of the type of intracranial lesion on outcome from severe head injury: a multicenter study using a new classification system. J Neurosurg 1982;56:26-32.

13. Gennarelli TA, Thibault LE. Biomechanics of acute subdural hematoma. J Trauma. 1982;22:680-686.

14. Hans JS. Head trauma evaluated magnetic resonance and computed tomography: a comparison. Radiology 1984;150:71-77.

15. Haselberger K, Rucher R, Auer LM. Prognosis after acute subdural and extradural hematoma. Acta Neurochir (Wien) 1988;90:111-116.

16. Hernesniemi J. Outcome following acute subdural hematoma. Acta Neurochir (Wien). 1979;49:191-198.

17. Howard MA, Gross AS, Dacey RG, Win HR. Acute subdural hematoma: an age dependent clinical entity. J Neurosurg 1989;71:858-863.

18. Janieson KG, Yelland JDN. Surgically treated traumatic subdural hematomas. J Neurosurg. 1968;29:13-23.

19. Klun B, Fettich M. Factors influencing the outcome in acute subdural hematoma: a review of 330 cases. Acta Neurochir (Wien). 1984;71:171-178.

20. Kotwica Z, Brzezi'nski J. Acute subdural hematoma in adults: an analysis of outcome in comatose patients. Acta Neurochir (Wien) 1993;121: 95-99.

21. Long DM. Aging in the nervous system. Neurosurgery 1985;17:348-354.

22. Mc Kissock W, Richardson A, Bloom WH. Acute subdural hematoma: a review of 389 cases. Lancet 1960;1:1365-1369.

23. Mc Laurin RL, Tutor FT. Acute subdural hematoma: review of 90 cases. J Neurosurg 1961;18:61-67.

24. Mendelow AD, Bullock R, Nath FP, Jinkins A, Kingman T, Teasdale GM: Experimental intracerebral haemorrage: intracranial pressure changes and cerebral blood flow. Intracranial pressure. Proceedings of the 6th International Symposium on Intracranial Pressure. Berlin: Springer-Verlag, 1986: 515-520.

25. Miller JD. Ischemic brain damage in a model of acute subdural hematoma. Neurosurgery 1990;27,433-439.

26. O'Brien PK, Norris JW, Tator CH. Acute subdural hematomas of arterial origin. J Neurosurg. 1974; 41: $435-439$.

27. Richards T, Hoff J. Factors affecting survival from acute subdural hematoma. Surgery 1974;75:253-258.

28. Schròder ML, Muizelaar JP, Kuta AJ. Documented reversal of global ischaemia immediately after removal of an acute subdural hematoma. J Neurosurg 1994;80:324-327.

29. Seelig JM. Traumatic acute subdural hematoma. Major mortality reduction in comatose patients treated within 4 hours. N Engl J Med 1981;304:1511-1518.

30. Shmidek HH. Operative neurosurgical techniques. Ed 3. Philadelphia: Saunders 1995:50-54.

31. Sonne NM, Tonnessen $H$. Course after surgery of subdural hematoma in alcohol abusers. Ugeskr Lauger $1994 ; 17: 156 ; 295-297$.

32. Stone JL. Acute subdural hematomas: progress in definition, clinical pathology, and therapy. Surg Neurol 1983;19:216-231.

33. Tallala A. Acute traumatic subdural hematoma: a review of 100 consecutive cases. J Trauma 1971;11:771-777.

34. Vielvoye GJ. Acute infratentorial subdural hematoma associated with a tom tentorium cerebelli in a 1 year old boy. Neuroradiology 1982;22:259-261.

35. Whittmore SR, Nieto-Sampiedro $M$, Needles DL et al. Neuronotrophic factors for mammalian brain neurons: injury inductions in neonatal, adult and aged rat brain. Dev Brain Res. 1985; 20: 169-178.

36. Wilberger JE Jr, Harris M, Diamond DL. Acute subdural hematoma : morbidity, mortality, and operative timing. J Neurosurg. 1991;74:212-218.

37. Youmans, JR. Neurological surgery. Ed 3. Philadelphia: Saunders 1990: 1962, 1977-1980, 2046-2049, 2077-2078, 2083-2085, 2204-2205. 